\title{
LIVER INVOLVEMENT IN INFECTIOUS MONONUCLEOSIS ${ }^{1}$
}

\author{
BY ALFRED S. EVANS \\ (From the Section of Preventive Medicine, Yale University School of Medicine, New Haven)
}

(Received for publication July 25, 1947)

The occurrence of hepatitis in the absence of jaundice in infectious mononucleosis has recently been emphasized. In 1946 Cohn and Lidman (1) published results of serial liver function studies carried out on 15 successive hospitalized patients with proved infectious mononucleosis in which there was neither clinical nor laboratory evidence of jaundice. All cases showed evidence of hepatic dysfunction as shown by abnormalities in more than 1 test. Carter and Maclagan (2) have studied 19 cases of infectious mononucleosis only one of which was jaundiced, and found elevated values for the thymol turbidity test in the serum of 58 per cent and a positive serum colloidal gold reaction in 95 per cent.

Since no serial study of the cephalin-cholesterol flocculation test in the serum of non-jaundiced infectious mononucleosis cases had been published, it was thought of interest to compare the results of this test in such serum with those of other measures of liver function.

\section{MATERIALS AND METHODS}

Serial determination of liver function tests were made on 19 consecutive cases of infectious mononucleosis without jaundice, 13 of which were hospitalized and 6 of which were ambulatory. ${ }^{2}$ Determination of sheep RBC agglutination was based on the method of Stuart (3) and agglutination in a final serum dilution of $1: 160$ or higher was considered significant. By this criteria, all except 2 cases had elevated heterophile antibody titers: one of these, at 1:80, showed guinea pig kidney and beef RBC absorption characteristics of the infectious mononucleosis antibody, and the other had a blood picture and a clinical course typical of infectious mononucleosis.

Cephalin-cholesterol flocculation tests were done by the technique of Hanger (4) and a reaction of $2+$ or more in 24 hours was considered abnormal. The thymol

1 Representing work done for the Virus and Rickettsial Disease Commission, Army Epidemiological Board, Office of the Surgeon General, U. S. Army, Washington, D. C.

2 Appreciation is expressed to Dr. John S. Hathaway and to the other physicians of the Yale University Department of Health for their kind cooperation in studying many of these patients. turbidity test was performed by Maclagan's method (5) and up to 4.0 units taken as normal. Serum bilirubin including the 1-minute determination followed the method of Malloy and Evelyn (6): normal values were taken as those up to 0.31 and $1.20 \mathrm{mgm}$. per cent for the 1-minute and total values, respectively. Four Bodansky units were accepted as the upper limit of normal for the alkaline serum phosphatase determination, utilizing his technique (7). In the few instances in which bromsulfalein excretion was measured, $5 \mathrm{mgm}$. per kilogram of body weight of the dye was injected and a value of 10 per cent or less was considered normal in the sample of serum drawn at the end of 30 minutes. Electrophoretic analysis of serum proteins ${ }^{8}$ was performed on 2 sera using the Tiselius apparatus, a barbiturate buffer of 0.1 ionic strength, and at a $\mathrm{pH}$ of 8.6.

\section{RESULTS}

The severity of the disease, the mononuclear percentage, the heterophile antibody titer, and the results of liver function tests, each shown according to the approximate day of the disease on which they were performed, are outlined in Table I, and in Figure 1 the percentage of cases whose sera showed abnormalities in liver function tests is compared with similar studies performed serially on the serum of patients with upper respiratory infections in which the diagnosis of infectious mononucleosis had been carefully excluded.

The cephalin-cholesterol flocculation test was abnormal in 95 per cent of the serum from patients with infectious mononucleosis and in 2 instances was altered before the heterophile antibody titer had reached a significant level. The 2 cases whose serum showed a normal flocculation reaction both had a mild illness during which they remained completely ambulatory. In contrast, none of the serum from 22 cases of upper respiratory infections, all of whom were ill enough to be hospitalized, demonstrated an abnormal cephalin flocculation test.

Elevated values for the thymol turbidity test

8 Electrophoretic analysis of serum proteins was kindly carried out by Dr. John Milne, Department of Internal Medicine, Yale University School of Medicine. 
were found in 68 per cent of the serum from patients with infectious mononucleosis and ranged from 4 to 10 units. Abnormalities in this test usually appeared later and were more transient than the changes in the cephalin flocculation reaction. One sample of serum from a patient with an upper respiratory infection showed a slightly increased value for the thymol turbidity test.

Forty-three per cent of the serum of infectious mononucleosis patients showed values for the alkaline phosphatase test from 4 to 11.5 Bodansky units. No serum from cases of infectious mononucleosis nor from those with respiratory conditions demonstrated significant increases in the total serum bilirubin determination, but slight increases in the 1-minute value above the arbitrary normal of $0.31 \mathrm{mgm}$. per cent were seen in both. Serial studies of bromsulfalein excretion were carried out in only 1 case of infectious mononucleosis and demonstrated a peak retention of $44 \mathrm{mgm}$. per cent in the 30-minute sample.

In Table II the results of analysis of serum proteins from a case of infectious mononucleosis without jaundice are compared with similar analysis of a serum from a jaundiced case, both of which were obtained approximately $1 \frac{1}{2}$ months after onset of illness. Marked increases in the percentages of beta and gamma globulin were observed in both.

TABLE I

Results of liver function studies in infectious mononucleosis cases without jaundice

\begin{tabular}{|c|c|c|c|c|c|c|c|c|c|c|c|}
\hline \multirow{2}{*}{ Case } & \multirow{2}{*}{$\begin{array}{l}\text { Day of } \\
\text { disease }\end{array}$} & \multirow{2}{*}{$\begin{array}{c}\text { Severity of } \\
\text { disease }\end{array}$} & \multirow{2}{*}{$\begin{array}{c}\text { Mono- } \\
\text { nuclears }\end{array}$} & \multirow{2}{*}{$\begin{array}{l}\text { Heterophile } \\
\text { antibody }\end{array}$} & \multirow{2}{*}{$\underset{\text { turb. }}{\text { Thymol }}$} & \multicolumn{2}{|c|}{ Cephalin flocculation } & \multicolumn{2}{|c|}{ Serum bilirubin } & \multirow{2}{*}{$\begin{array}{c}\text { Alkaline } \\
\text { phospha- } \\
\text { tase }\end{array}$} & \multirow{2}{*}{$\underset{(30-\text { min. })}{\text { BSP }}$} \\
\hline & & & & & & 24 & 48 & 1 Min. & Total & & \\
\hline 1 & $\begin{array}{r}9 \\
11 \\
15 \\
29 \\
32 \\
45\end{array}$ & Severe & $\begin{array}{c}\text { per cent } \\
23 \\
68 \\
53\end{array}$ & $\begin{array}{c}\text { titer } \\
1: 1280 \\
1: 1280 \\
1: 2560 \\
1: 640 \\
1: 320 \\
1: 160\end{array}$ & $\begin{array}{c}\text { units } \\
2.5 \\
2.0 \\
2.0 \\
4.5 \\
6.0 \\
4.5\end{array}$ & $\begin{array}{l}\mathbf{0} \\
\mathbf{3} \\
\mathbf{3} \\
\mathbf{3} \\
\mathbf{3} \\
\mathbf{3}\end{array}$ & $\begin{array}{l}1 \\
4 \\
3 \\
3 \\
3 \\
3\end{array}$ & $\begin{array}{l}\text { mgm. } \\
0.07 \\
0.37 \\
0.51 \\
0.41 \\
0.61 \\
0.34\end{array}$ & $\begin{array}{c}\text { per cent } \\
0.48 \\
1.02 \\
0.94 \\
0.88 \\
1.63 \\
0.61\end{array}$ & $\begin{array}{c}\begin{array}{c}\text { Bodansky } \\
\text { units }\end{array} \\
\\
5.50 \\
5.35 \\
4.32 \\
2.87 \\
2.08\end{array}$ & per cent \\
\hline 2 & $\begin{array}{l}10 \\
14 \\
30\end{array}$ & Severe & $\begin{array}{l}80 \\
72\end{array}$ & $\begin{array}{l}1: 2560 \\
1: 1280 \\
1: 320\end{array}$ & $\begin{array}{l}5.5 \\
5.5 \\
5.0\end{array}$ & $\begin{array}{l}3 \\
3 \\
2\end{array}$ & $\begin{array}{l}4 \\
4 \\
3\end{array}$ & $\begin{array}{l}0.03 \\
0.48\end{array}$ & $\begin{array}{l}0.75 \\
0.88\end{array}$ & $\begin{array}{l}2.65 \\
3.33\end{array}$ & \\
\hline 3 & $\begin{array}{r}2 \\
11 \\
15 \\
21\end{array}$ & Severe & 53 & $\begin{array}{l}1: 40 \\
1: 2560 \\
1: 1280 \\
1: 1280\end{array}$ & $\begin{array}{l}2.0 \\
4.5 \\
3.0 \\
7.5\end{array}$ & $\begin{array}{l}3 \\
3 \\
3 \\
4\end{array}$ & $\begin{array}{l}4 \\
4 \\
4 \\
4\end{array}$ & $\begin{array}{l}0.10 \\
0.24 \\
0.20 \\
0.20\end{array}$ & $\begin{array}{l}1.20 \\
1.22 \\
0.75 \\
1.09\end{array}$ & $\begin{array}{l}1.43 \\
2.73 \\
2.78\end{array}$ & 8.4 \\
\hline 4 & $\begin{array}{l}5 \\
9\end{array}$ & Severe & 49 & $\begin{array}{l}1: 640 \\
1: 160\end{array}$ & $\begin{array}{l}4.5 \\
3.0\end{array}$ & $\begin{array}{l}3 \\
3\end{array}$ & $\begin{array}{l}4 \\
4\end{array}$ & $\begin{array}{l}0.07 \\
0.27\end{array}$ & $\begin{array}{l}0.27 \\
0.61\end{array}$ & 5.95 & 2.0 \\
\hline 5 & $\begin{array}{l}30 \\
38 \\
44 \\
48\end{array}$ & Severe & $\begin{array}{l}61 \\
68\end{array}$ & $\begin{array}{l}1: 1280 \\
1: 1280 \\
1: 1280 \\
1: 1280\end{array}$ & $\begin{array}{l}2.0 \\
4.5 \\
5.5\end{array}$ & $\begin{array}{l}3 \\
3 \\
1 \\
\pm\end{array}$ & $\begin{array}{l}4 \\
4 \\
2 \\
1\end{array}$ & $\begin{array}{l}0.07 \\
0.07\end{array}$ & $\begin{array}{l}0.27 \\
0.41\end{array}$ & & 0 \\
\hline 6 & $\begin{array}{r}7 \\
10 \\
16 \\
41\end{array}$ & Severe & $\begin{array}{l}54 \\
70\end{array}$ & $\begin{array}{l}1: 80 \\
1: 640 \\
1: 1280 \\
1: 640\end{array}$ & $\begin{array}{l}2.0 \\
2.5 \\
6.0 \\
1.0\end{array}$ & $\begin{array}{l}2 \\
4 \\
3 \\
4\end{array}$ & $\begin{array}{l}3 \\
4 \\
3 \\
4\end{array}$ & $\begin{array}{l}0.34 \\
0.10 \\
0.44 \\
0.07\end{array}$ & $\begin{array}{l}0.95 \\
0.28 \\
1.16 \\
1.16\end{array}$ & $\begin{array}{r}4.23 \\
11.50 \\
3.75\end{array}$ & $\begin{array}{l}18 \\
44 \\
13\end{array}$ \\
\hline 7 & $\begin{array}{r}8 \\
15\end{array}$ & Severe & & $\begin{array}{l}1: 640 \\
1: 640\end{array}$ & $\begin{array}{r}6.0 \\
10.0\end{array}$ & $\begin{array}{l}2 \\
4\end{array}$ & $\begin{array}{l}4 \\
4\end{array}$ & 0.10 & 0.28 & 2.18 & 1.5 \\
\hline 8 & $\begin{array}{r}5 \\
8 \\
17\end{array}$ & Moderate & 65 & $\begin{array}{l}1: 160 \\
1: 640 \\
1: 1280\end{array}$ & $\begin{array}{l}2.5 \\
3.5 \\
6.0\end{array}$ & $\begin{array}{l}3 \\
4\end{array}$ & $\begin{array}{l}4 \\
4\end{array}$ & $\begin{array}{l}0.14 \\
0.14 \\
0.37\end{array}$ & $\begin{array}{l}0.34 \\
0.38 \\
1.09\end{array}$ & 3.10 & \\
\hline 9 & $\begin{array}{l}12 \\
53\end{array}$ & Moderate & 61 & $\begin{array}{l}1: 640 \\
1: 80\end{array}$ & $\begin{array}{l}3.0 \\
2.0\end{array}$ & $\begin{array}{l}2 \\
0\end{array}$ & $\begin{array}{l}3 \\
0\end{array}$ & $\begin{array}{l}0.58 \\
0.10\end{array}$ & $\begin{array}{l}0.98 \\
0.34\end{array}$ & 9.77 & \\
\hline 10 & $\begin{array}{r}8 \\
11\end{array}$ & Moderate & $\begin{array}{l}89 \\
77\end{array}$ & $\begin{array}{l}1: 640 \\
1: 640\end{array}$ & $\begin{array}{l}5.0 \\
2.5\end{array}$ & $\begin{array}{l}4 \\
3\end{array}$ & $\begin{array}{l}4 \\
4\end{array}$ & $\begin{array}{l}0.24 \\
0.07\end{array}$ & $\begin{array}{l}0.61 \\
0.41\end{array}$ & $\begin{array}{l}7.75 \\
2.64\end{array}$ & \\
\hline
\end{tabular}


TABLE I-Continued

\begin{tabular}{|c|c|c|c|c|c|c|c|c|c|c|c|}
\hline \multirow{2}{*}{ Case } & \multirow{2}{*}{$\begin{array}{l}\text { Day of } \\
\text { disease }\end{array}$} & \multirow{2}{*}{$\begin{array}{l}\text { Severity of } \\
\text { disease }\end{array}$} & \multirow{2}{*}{$\begin{array}{c}\text { Mono- } \\
\text { nuclears }\end{array}$} & \multirow{2}{*}{$\begin{array}{l}\text { Heterophile } \\
\text { antibody }\end{array}$} & \multirow{2}{*}{$\begin{array}{l}\text { Thymol } \\
\text { turb. }\end{array}$} & \multicolumn{2}{|c|}{ Cephalin flocculation } & \multicolumn{2}{|c|}{ Serum bilirubin } & \multirow{2}{*}{$\begin{array}{c}\text { Alkaline } \\
\text { phospha- } \\
\text { tase }\end{array}$} & \multirow{2}{*}{$\begin{array}{c}\text { BSP } \\
(30-\text { min. })\end{array}$} \\
\hline & & & & & & 24 & 48 & $1 \mathrm{Min}$. & Total & & \\
\hline 11 & 17 & Moderate & $\begin{array}{c}\text { per cent } \\
91\end{array}$ & $\begin{array}{c}\text { titer } \\
1: 1280\end{array}$ & $\begin{array}{c}\text { units } \\
7.0\end{array}$ & 4 & 4 & $\begin{array}{l}m g m . \\
0.07\end{array}$ & $\begin{array}{c}\text { per cent } \\
0.42\end{array}$ & $\begin{array}{c}\text { Bodansky } \\
\text { units }\end{array}$ & per cent \\
\hline 12 & 10 & Moderate & & $1: 1280$ & 5.0 & 3 & 4 & 0.17 & 0.48 & 2.95 & \\
\hline 13 & $\begin{array}{r}7 \\
13\end{array}$ & Moderate & $\begin{array}{l}70 \\
54\end{array}$ & $\begin{array}{l}1: 40 \\
1: 80\end{array}$ & $\begin{array}{l}3.0 \\
3.0\end{array}$ & $\begin{array}{l}2 \\
0\end{array}$ & $\begin{array}{l}3 \\
1\end{array}$ & $\begin{array}{l}0.68 \\
0.68\end{array}$ & $\begin{array}{l}0.24 \\
0.14\end{array}$ & & \\
\hline 14 & $\begin{array}{r}6 \\
12 \\
22\end{array}$ & Moderate & & $\begin{array}{l}1: 640 \\
1: 2560 \\
1: 640\end{array}$ & $\begin{array}{l}3.0 \\
5.0 \\
2.0\end{array}$ & $\begin{array}{l}2 \\
3 \\
3\end{array}$ & $\begin{array}{l}3 \\
4 \\
3\end{array}$ & $\begin{array}{l}.07 \\
.31 \\
.31\end{array}$ & $\begin{array}{l}0.31 \\
0.75 \\
1.22\end{array}$ & $\begin{array}{l}3.75 \\
2.63 \\
5.45\end{array}$ & \\
\hline 15 & $\begin{array}{r}4 \\
17 \\
66\end{array}$ & Mild & $\begin{array}{l}67 \\
42\end{array}$ & $\begin{array}{l}1: 640 \\
1: 640 \\
1: 40\end{array}$ & $\begin{array}{l}4.0 \\
3.0\end{array}$ & $\begin{array}{l}3 \\
0 \\
0\end{array}$ & $\begin{array}{l}4 \\
0 \\
1\end{array}$ & $\begin{array}{l}0.14 \\
0.34 \\
0.07\end{array}$ & $\begin{array}{l}0.38 \\
0.82 \\
0.20\end{array}$ & & \\
\hline 16 & $\begin{array}{r}6 \\
28\end{array}$ & Mild & 63 & $\begin{array}{l}1: 640 \\
1: 640\end{array}$ & $\begin{array}{l}3.0 \\
2.0\end{array}$ & $\begin{array}{l}3 \\
3\end{array}$ & $\begin{array}{l}4 \\
4\end{array}$ & $\begin{array}{l}0.07 \\
0.07\end{array}$ & $\begin{array}{l}0.17 \\
0.28\end{array}$ & $\begin{array}{l}3.03 \\
1.90\end{array}$ & \\
\hline 17 & $\begin{array}{r}3 \\
4 \\
8 \\
20\end{array}$ & Mild & $\begin{array}{l}48 \\
69\end{array}$ & $\begin{array}{l}\text { Under } 1: 5 \\
1: 5 \\
1: 10\end{array}$ & $\begin{array}{l}1.0 \\
1.0 \\
1.5\end{array}$ & $\begin{array}{l}3 \\
2 \\
2\end{array}$ & $\begin{array}{l}4 \\
4 \\
3\end{array}$ & $\begin{array}{l}0.10 \\
\\
0.03 \\
0.10\end{array}$ & $\begin{array}{l}0.61 \\
\\
0.27 \\
0.54\end{array}$ & 1.30 & \\
\hline 18 & $\begin{array}{l}4 \\
8\end{array}$ & Mild & 50 & $\begin{array}{l}1: 640 \\
1: 160\end{array}$ & $\begin{array}{l}1.5 \\
3.0\end{array}$ & $\begin{array}{l}0 \\
0\end{array}$ & $\begin{array}{l}0 \\
0\end{array}$ & $\begin{array}{l}0.10 \\
0.07\end{array}$ & $\begin{array}{l}0.48 \\
0.34\end{array}$ & 2.78 & \\
\hline 19 & 10 & Mild & & $1: 1280$ & 4.5 & 0 & 2 & 0.27 & 0.68 & & \\
\hline
\end{tabular}

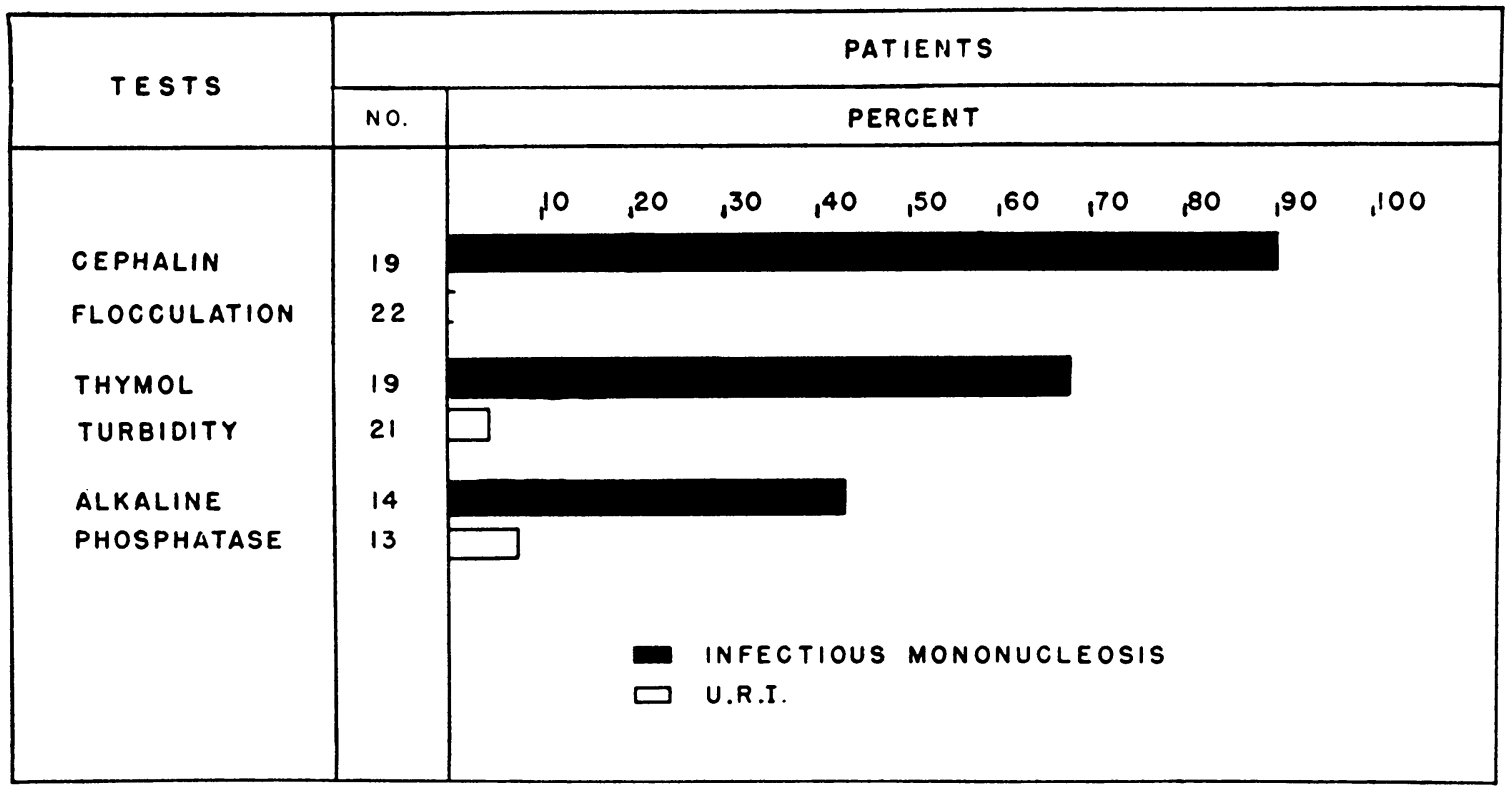

Fig. 1. Percentage of Patients with Infectious Mononucleosis Whose Sera Showed Abnormalities in Liver Function Tests as Compared with Patients with Upper Respiratory Infections. No Serum from a Patient with an U.R.I. Showed an Abnormal Cephalin Flocculation Test 
TABLE II

Results of electrophoretic analysis of serum proteins from

2 convalescent infectious mononucleosis cases expressed as per cent of total protein

\begin{tabular}{l|c|c|c|c}
\hline \hline \multicolumn{1}{c|}{ Condition } & Albumin & \multicolumn{3}{|c}{ Globulin } \\
\cline { 2 - 4 } & & Alpha & Beta & Gamma \\
\hline $\begin{array}{l}\text { Inf. mononucleosis with } \\
\text { jaundice } \\
\begin{array}{l}\text { Inf. mononucleosis without } \\
\text { jaundice }\end{array}\end{array}$ & 52.5 & 4.1 & 22.7 & 20.7 \\
\begin{tabular}{l} 
Normal \\
\hline
\end{tabular} & 65.0 & 4.9 & 27.3 & 20.7 \\
\hline
\end{tabular}

\section{DISCUSSION}

It has been pointed out in this paper that a demonstrable hepatitis occurs in many cases of infectious mononucleosis without jaundice. These results are in accord with previous investigations $(1,2)$. In addition the results of this study suggest that the cephalin-cholesterol flocculation reaction is a more sensitive indicator of this hepatic dysfunction than the thymol turbidity test and may be used in differentiating such cases from uncomplicated upper respiratory infections.

Histopathological evidence of liver involvement in non-jaundiced infectious mononucleosis cases has been reported from both punch biopsy studies $(8,9)$ and from autopsy reports (10 to 13$)$. These consist of infiltrative mononuclear lesions frequently associated with destruction and disappearance of the liver cells, and the microscopic picture has been said to resemble early infectious hepatitis ( 8 to 10 ). Similar morphological studies carried out on the livers of patients with infectious mononucleosis complicated by jaundice $(14,15)$ have also demonstrated lesions closely comparable to infectious hepatitis.

The similarities between the hepatic involvement in infectious mononucleosis and infectious hepatitis are frequently reflected in the clinical picture of these diseases. Indeed, cases of infectious mononucleosis with jaundice frequently cause much difficulty in differential diagnosis from cases of infectious hepatitis (16). The hematological picture of the early phase of both naturally occurring (17 to 20 ) and experimentally induced (21) infectious hepatitis may closely simulate that of infectious mononucleosis and as many as 60 per cent of the lymphocytes have been described as atypical in infectious hepatitis (18). Increases of the characteristic heterophile antibody of infec- tious mononucleosis have not been described in infectious hepatitis (18), although Eaton and his associates (22) have found a heterogenetic antibody in a few such cases that could be differentiated only by absorption tests. Since increases of the heterophile antibody may not always be demonstrable in the serum of patients with infectious mononucleosis, occasional cases of these 2 diseases may be nearly indistinguishable.

\section{SUMM ARY AND CONCLUSIONS}

1. The results of serial liver function tests carried out in this laboratory on 19 cases of infectious mononucleosis without jaundice are presented and indicate that demonstrable hepatic impairment occurs in many such cases.

2. The cephalin-cholesterol flocculation test may be a more sensitive indicator of this disturbance than the thymol turbidity test.

3. Use of the cephalin-cholesterol flocculation and the thymol turbidity tests may be helpful in distinguishing infectious mononucleosis from uncomplicated upper respiratory infections.

4. Relations between infectious mononucleosis and infectious hepatitis are briefly discussed.

Since the completion of this paper, the results of a similar study have been published by Q. B. DeMarsh and H. L. Alt (23) reporting abnormalities in the cephalincholesterol flocculation and/or the sulf-bromophthalein tests in 19 cases of infectious mononucleosis without clinical jaundice on whom serial liver function tests were performed. There has also been a report by Gall (24) recording similar findings in 34 cases.

\section{BIBLIOGRAPHY}

1. Cohn, C., and Lidman, B. I., Hepatitis without jaundice in infectious mononucleosis. J. Clin. Invest., 1946, 25, 145.

2. Carter, A. B., and Maclagan, N. F., Some observations on liver function tests in diseases not primarily hepatic. Brit. Med. J., 1946, 2, 80.

3. American Public Health Association, Diagnostic Procedures and Reagents. Am. Public Health Assoc., New York, 1945.

4. Hanger, F. M., Serological differentiation of obstructive from hepatogenous jaundice by flocculation of cephalin-cholesterol emulsions. J. Clin. Invest., 1939, 18, 261.

5. Maclagan, N. F., Thymol turbidity test; new indicator of liver dysfunction. Brit. J. Exp. Path., 1944, 25, 234.

6. Malloy, H. T., and Evelyn, K. A., The determination of bilirubin with the photoelectric colorimeter. J. Biol. Chem., 1937, 119, 481. 
7. Bodansky, A., Notes on the determination of serum inorganic phosphate and serum phosphatase activity. J. Biol. Chem., 1937, 120, 167.

8. Davis, J. S., MacFee, W., Wright, M., and Allyn, R., Rupture of the spleen in infectious mononucleosis. Lancet, 1945, 2, 72.

9. Van Beek, S. I., and Haex, A. J. Ch., Aspirationbiopsy of the liver in infectious mononucleosis and in Besnier-Boeck-Schaumann's disease. Acta. med. Scandinav., 1943, 113, 125.

10. Fisher, J. H., Visceral lesions of acute infectious mononucleosis : a report of 2 cases with fatal spontaneous rupture of the spleen. Am. J. Path., 1946, 42, 651.

11. Ziegler, E. E., Infectious mononucleosis : report of a fatal case with autopsy. Arch. Path., 1944, 37, 196.

12. Ricker, W., Blumberg, A., Peters, C. H., and Widerman, A., The association of the Guillain-Barré syndrome with infectious mononucleosis with a report of 2 fatal cases. Blood, 1947, 3, 217.

13. Allen, F. H., Jr., and Kellner, A., Infectious mononucleosis, an autopsy report. Am. J. Path., 1947, 23, 463.

14. Kilham, L., and Steigman, A. J., Infectious mononucleosis. Lancet, 1942, 2, 452.

15. Bang, J., and Wanscher, O., The histopathology of the liver in infectious mononucleosis complicated by jaundice. Acta. med. Scandinav., 1945, 120, 437.
16. Wechsler, H. F., Rosenblum, A. H., and Sills, C. T., Infectious mononucleosis: report of an epidemic in an army post, part 1. Ann. Int. Med., 1946, 25, 113.

17. Jones, C. M., and Minot, G. R., Observations on excretion and retention of bile pigments, and on blood. Infectious (catarrhal) jaundice; an attempt to establish a clinical entity. Boston M. and S. J., 1923, 189, 531.

18. Barker, M. H., Capps, R. B., and Allen, F. W., Acute infectious hepatitis in the Mediterranean theatre, including acute hepatitis without jaundice. J. A. M. A., 1945, 128, 997.

19. Thewlis, E., and Middleton, W. S., The leucocytic picture in catarrhal jaundice (cholangitis). Am. J. M. Sc., 1925, 169, 59.

20. Horstmann, D. M., Havens, W. P., Jr., and Deutsch; $\mathrm{J}$., Infectious hepatitis in childhood. J. Pediat., 1947, 30, 381.

21. Havens, W. P., Jr., and Marck, R. E., The leukocytic response of patients with experimentally induced infectious hepatitis. Am. J. M. Sc., 1946, 212, 129.

22. Eaton, M. D., Murphy, W. D., and Hanford, V. L., Heterogenetic antibodies in acute hepatitis. J. Exper. Med., 1944, 79, 539.

23. DeMarsh, Q. B., and Alt, H. L., Hepatitis without jaundice in infectious mononucleosis. Arch. Int. Med., 1947, 80, 257.

24. Gall, E. A., Serum phosphatase and other tests of liver function in infectious mononucleosis. Amer. J. Clin. Path., 1947, 17, 529. 